\title{
Editorial
}

\section{Sentiment Analysis for Social Media}

\author{
Carlos A. Iglesias ${ }^{1, *,+} \mathbb{D}$ and Antonio Moreno ${ }^{2,+} \mathbb{D}$ \\ 1 Intelligent Systems Group, ETSI Telecomunicación, Avda. Complutense 30, 28040 Madrid, Spain \\ 2 Intelligent Technologies for Advance Knowledge Acquisition (ITAKA) Group, \\ Escola Tècnica Superior d'Enginyeria, Departament d'Enginyeria Informàtica i Matemàtiques, \\ Universitat Rovira i Virgili, 43007 Tarragona, Spain; antonio.moreno@urv.cat \\ * Correspondence: carlosangel.iglesias@upm.es; Tel.: +34-910671900 \\ + These authors contributed equally to this work.
}

Received: 31 October 2019; Accepted: 19 November 2019; Published: 22 November 2019

\begin{abstract}
Sentiment analysis has become a key technology to gain insight from social networks. The field has reached a level of maturity that paves the way for its exploitation in many different fields such as marketing, health, banking or politics. The latest technological advancements, such as deep learning techniques, have solved some of the traditional challenges in the area caused by the scarcity of lexical resources. In this Special Issue, different approaches that advance this discipline are presented. The contributed articles belong to two broad groups: technological contributions and applications.
\end{abstract}

Keywords: sentiment analysis; emotion analysis; social media; affect computing

\section{Introduction}

Sentiment analysis technologies enable the automatic analysis of the information distributed through social media to identify the polarity of posted opinions [1]. These technologies have been extended in the last years to analyze other aspects, such as the stance of a user towards a topic [2] or the users' emotions [3], even combining text analytics with other inputs, including multimedia analysis [4] or social network analysis [5].

This Special Issue "Sentiment Analysis for Social Media" aims to reflect recent developments in sentiment analysis and to present new advances in sentiment analysis that enable the development of future sentiment analysis and social media monitoring methods. The following sections detail the selected works in the development of new techniques as well as new applications.

\section{New Paths in Sentiment Analysis on Social Media}

Traditionally, sentiment analysis has focused on text analysis using Natural Language Processing and feature-based Machine Learning techniques. The advances in disciplines such as Big Data and Deep Learning technologies have impacted and benefited the evolution of the field. This Special Issue includes four works that propose novel techniques.

In the first work, titled "An Improved Study of Multilevel Semantic Network Visualization for Analyzing Sentiment Word of Movie Review Data" [6], Ha et al. propose a method for sentiment visualization in massive social media. For this purpose, they design a multi-level sentiment network visualization mechanism based on emotional words in the movie review domain. They propose three visualization methods: a heatmap visualization of the semantic words of every node, a two-dimensional scaling map of semantic word data, and a constellation visualization using asterism images for each cluster of the network. The proposed visualizations have been used as a recommender system that suggest movies with similar emotions to the previously watched ones. This novel idea of recommending contents based on similar emotional patterns can be applied to other social networks. 
In the second contribution, titled "Sentiment Classification Using Convolutional Neural Networks" [7], Kim and Jeong deal with the problem of textual sentiment classification. They propose a Convolutional Neural Network (CNN) model consisting of an embedding layer, two convolutional layers, a pooling layer, and a fully-connected layer. The model is evaluated in three datasets (movie review data, customer review data and Stanford Sentiment Treebank data) and compared with traditional Machine Learning models and state of the art Deep Learning models. Their main conclusion is that the use of consecutive convolutional layers is effective for relatively long texts.

In the third work, titled "Sentiment-Aware Word Embedding for Emotion Classification" [8], Mao et al. suggest the use of a sentiment-aware word embedding for improving emotional analysis. The proposed method builds a hybrid representation that combines emotional word embeddings based on an emotional lexicon with semantic word embeddings based on Word2Vec [9]. They use the emotional lexicon DUTIR, which is a Chinese ontology resource collated and labeled by the Dalian University of Technology Information Retrieval Laboratory [10]. This resource annotates lexicon entries with a model of seven emotions (happiness, trust, anger, sadness, fear, disgust and surprise). The evaluation is done with data from Weibo, a popular Chinese social networking site. The paper evaluates two methods (direct combination and addition) for building the hybrid representation in several datasets. They conclude that the experiments prove that the use of hybrid word vectors is effective for supervised emotion classification, improving significantly the classification accuracy.

Finally, in the fourth theoretical contribution, titled "A Deep Learning-Based Approach for Multi-Label Emotion Classification in Tweets" [11], Jabreel and Moreno address the problem of multi-class emotion classification based on Deep Learning techniques. The most popular approach for this problem is to transform it into multiple binary classification problems, one for each emotion class. This paper proposes a new transformation approach, so-called $x y$-pair-set, that transforms the original problem into just one binary classification problem. The transformation problem is solved with a Deep Learning-based system, so-called BNet. This system consists of three modules: an embedding module that uses three embedding models and an attention function, an encoding module based on Recurrent Neural Networks (RNNs), and a classification module that uses two feed-forward layers with the $R e L U$ activation function followed by a sigmoid unit. The system is evaluated using the dataset "Affect in Tweets" of SemEval-2019 Task 1 [2], and it outperformed the state of the art systems.

\section{Applications of Sentiment Analysis in Social Media}

The wide range of applications of sentiment analysis has fostered its evolution. Sentiment analysis techniques have enabled to make sense of big social media data to make more informed decisions and understand social events, product marketings or political events. Four works selected in this Special Issue deal with the application of sentiment analysis for improving health insurances, understanding AIDS patients, e-commerce user profiling and cyberagression detection.

In the first work, titled "Using Social Media to Identify Consumers' Sentiments towards Attributes of Health Insurance during Enrollment Season" [12], van den Broek-Altenburg and Atherly aim at understanding the consumers' sentiments towards health insurances. For this purpose, they mined Twitter discussions and analyzed them using a dictionary-based approach using the NRC Emotion Lexicon [13], which provides for each word its polarity as well as its related emotion (anger, anticipation, disgust, fear, joy, sadness, surprise and trust). The main finding of this study is that consumers are worried about providers networks, prescription drug benefits and political preferences. In addition, consumers trust medical providers but fear unexpected events. These results suggest that more research is needed to understand the origin of the sentiments that drive consumers so that insurers can provide better insurance plans.

In the second contribution, titled "Gender Classification Using Sentiment Analysis and Deep Learning in a Health Web Forum" [14], Park and Woo deal also with the application of sentiment analysis techniques to health-related topics. In particular, they apply sentiment analysis for identifying gender in health forums based on Deep Learning techniques. The authors analyze messages from 
an AIDS-related bulletin board from HealthBoard.com and evaluate both traditional and Deep Learning techniques for gender classification.

In the third approach [15], titled "Personality or Value: A Comparative Study of Psychographic Segmentation Based on an Online Review Enhanced Recommender System", Liu et al. analyze the predictive and explanatory capability of psychographic characteristics in e-commerce user preferences. For this purpose, they construct a pychographic lexicon based on seed words provided by psycholinguistics that are expanded using synonyms from WordNet [16], resulting in positive and negative lexicons for two psychographic models, Schwartz Value Survey (SVS) [17] and Big Five Factor (BFF) [18]. Then they construct word embeddings using Word2Vec [9] and extend the corpus with word embeddings from an Amazon corpus [19]. Finally, they incorporate the lexicons in a deep neural network-based recommender system to predict the users' online purchasing behaviour. They also evaluate customer segmentation based on BDSCAN clustering [20], but this does not provide a significant improvement. The main insight of this research is that psychographic variables improve the explanatory power of e-consumer preferences, but their prediction capability is not significant.

Finally, in the fourth work [21], titled "Classification of Cyber-Aggression Cases Applying Machine Learning", Gutiérrez-Esparza et al. deal with the detection of cyberagression. They build and label a corpus of cyberagression news from Facebook in Latinamerica and develop a classification model based on Machine Learning techniques. The developed corpus can foster research in this field, given the scarcity of lexical resources in languages different from English.

\section{Conclusions}

The diversity of approaches of the articles included in this Special Issue shows the great interest and dynamism of this field. Moreover, this Special Issue of Applied Sciences contributes to provide a good overview of some of the main areas of research in this field.

Funding: This research received no external funding.

Acknowledgments: The Guest Editors would like to thank all the authors that have participated in this Special Issue and also the reference contact in MDPI, Nyssa Yuan, for all the support and work dedicated to the success of this Special Issue.

Conflicts of Interest: The authors declare no conflict of interest.

\section{References}

1. Liu, B. Sentiment analysis and opinion mining. Synth. Lect. Hum. Lang. Technol. 2012, 5, 1-167.

2. Mohammad, S.; Bravo-Marquez, F.; Salameh, M.; Kiritchenko, S. Semeval-2018 task 1: Affect in tweets. In Proceedings of the 12th International Workshop on Semantic Evaluation, New Orleans, LA, USA, 5-6 June 2018; pp. 1-17.

3. Cambria, E.; Poria, S.; Hussain, A.; Liu, B. Computational Intelligence for Affective Computing and Sentiment Analysis [Guest Editorial]. IEEE Comput. Intell. Mag. 2019, 14, $16-17$.

4. Li, Z.; Fan, Y.; Jiang, B.; Lei, T.; Liu, W. A survey on sentiment analysis and opinion mining for social multimedia. Multimed. Tools Appl. 2019, 78, 6939-6967.

5. Sánchez-Rada, J.F.; Iglesias, C.A. Social context in sentiment analysis: Formal definition, overview of current trends and framework for comparison. Inf. Fusion 2019, 52, 344-356.

6. Ha, H.; Han, H.; Mun, S.; Bae, S.; Lee, J.; Lee, K. An Improved Study of Multilevel Semantic Network Visualization for Analyzing Sentiment Word of Movie Review Data. Appl. Sci. 2019, 9, 2419. [CrossRef]

7. Kim, H.; Jeong, Y.S. Sentiment Classification Using Convolutional Neural Networks. Appl. Sci. 2019, 9, 2347. [CrossRef]

8. Mao, X.; Chang, S.; Shi, J.; Li, F.; Shi, R. Sentiment-Aware Word Embedding for Emotion Classification. Appl. Sci. 2019, 9, 1334. [CrossRef]

9. Mikolov, T.; Chen, K.; Corrado, G.; Dean, J. Efficient estimation of word representations in vector space. arXiv 2013, arXiv:1301.3781. 
10. Chen, J. The Construction and Application of Chinese Emotion Word Ontology. Master's Thesis, Dailian University of Technology, Dalian, China, 2008.

11. Jabreel, M.; Moreno, A. A Deep Learning-Based Approach for Multi-Label Emotion Classification in Tweets. Appl. Sci. 2019, 9, 1123. [CrossRef]

12. Van den Broek-Altenburg, E.M.; Atherly, A.J. Using Social Media to Identify Consumers' Sentiments towards Attributes of Health Insurance during Enrollment Season. Appl. Sci. 2019, 9, 2035. [CrossRef]

13. Mohammad, S.M.; Kiritchenko, S.; Zhu, X. NRC-Canada: Building the state-of-the-art in sentiment analysis of tweets. arXiv 2013, arXiv:1308.6242.

14. Park, S.; Woo, J. Gender Classification Using Sentiment Analysis and Deep Learning in a Health Web Forum. Appl. Sci. 2019, 9, 1249. [CrossRef]

15. Liu, H.; Huang, Y.; Wang, Z.; Liu, K.; Hu, X.; Wang, W. Personality or Value: A Comparative Study of Psychographic Segmentation Based on an Online Review Enhanced Recommender System. Appl. Sci. 2019, 9, 1992. [CrossRef]

16. Miller, G.A. WordNet: A lexical database for English. Commun. ACM 1995, 38, 39-41. [CrossRef]

17. Sagiv, L.; Schwartz, S.H. Cultural values in organisations: Insights for Europe. Eur. J. Int. Manag. 2007, 1, 176-190. [CrossRef]

18. McCrae, R.R.; Costa, P.T., Jr. The five-factor theory of personality. In Handbook of Personality: Theory and Research; The Guilford Press: New York, NY, USA, 2008; pp. 159-181.

19. McAuley, J.; Targett, C.; Shi, Q.; Van Den Hengel, A. Image-based recommendations on styles and substitutes. In Proceedings of the 38th International ACM SIGIR Conference on Research and Development in Information Retrieval, Santiago, Chile, 9-13 August 2015; pp. 43-52.

20. Ester, M.; Kriegel, H.P.; Sander, J.; Xu, X.. A density-based algorithm for discovering clusters in large spatial databases with noise. In KDD-96 Proceddings; AAAI Press: Portland, OR, USA, 1996; pp. 226-231.

21. Gutiérrez-Esparza, G.O.; Vallejo-Allende, M.; Hernández-Torruco, J. Classification of Cyber-Aggression Cases Applying Machine Learning. Appl. Sci. 2019, 9, 1828. [CrossRef]

(C) 2019 by the authors. Licensee MDPI, Basel, Switzerland. This article is an open access article distributed under the terms and conditions of the Creative Commons Attribution (CC BY) license (http://creativecommons.org/licenses/by/4.0/). 